\title{
Prediction of MoRFs based on sequence properties and convolutional neural networks
}

\author{
Hao He, Yatong Zhou* ${ }^{*}$, Yue Chi and Jingfei He
}

\author{
* Correspondence: zyt@hebut.edu. \\ $\mathrm{cn}$ \\ School of Electronic and \\ Information Engineering, Hebei \\ University of Technology, Tianjin, \\ China
}

\begin{abstract}
Background: Intrinsically disordered proteins possess flexible 3-D structures, which makes them play an important role in a variety of biological functions. Molecular recognition features (MoRFs) act as an important type of functional regions, which are located within longer intrinsically disordered regions and undergo disorder-toorder transitions upon binding their interaction partners.

Results: We develop a method, MoRF ${ }_{C N N}$, to predict MoRFs based on sequence properties and convolutional neural networks (CNNs). The sequence properties contain structural and physicochemical properties which are used to describe the differences between MoRFs and non-MoRFs. Especially, to highlight the correlation between the target residue and adjacent residues, three windows are selected to preprocess the selected properties. After that, these calculated properties are combined into the feature matrix to predict MoRFs through the constructed CNN. Comparing with other existing methods, MoRF $\mathrm{CNN}_{\mathrm{N}}$ obtains better performance.
\end{abstract}

Conclusions: MoRF ${ }_{C N N}$ is a new individual MoRFs prediction method which just uses protein sequence properties without evolutionary information. The simulation results show that MoRF ${ }_{C N N}$ is effective and competitive.

Keywords: Molecular recognition features, Intrinsically disordered proteins, Prediction, Convolutional neural network

\section{Background}

Recently, it has been recognized that many proteins, or regions of proteins, lack stable 3-D structures under apparently native conditions [1]. These proteins are called intrinsically disordered proteins (IDPs). Despite the lack of stable 3-D structures, IDPs have been confirmed to perform a variety of important biological functions, and thus are correlated with some diseases such as cancer and Alzheimer's disease [2]. Molecular recognition features (MoRFs) act as an important type of functional region in IDPs. MoRFs permit interaction with structured partner proteins and can undergo disorderto-order transitions upon interaction [3]. They generally vary in size and are up to 70 residues long, which are located within longer intrinsically disordered regions [4].

(c) The Author(s). 2021 Open Access This article is licensed under a Creative Commons Attribution 4.0 International License, which permits use, sharing, adaptation, distribution and reproduction in any medium or format, as long as you give appropriate credit to the original author(s) and the source, provide a link to the Creative Commons licence, and indicate if changes were made. The images or other third party material in this article are included in the article's Creative Commons licence, unless indicated otherwise in a credit line to the material. If material is not included in the article's Creative Commons licence and your intended use is not permitted by statutory regulation or exceeds the permitted use, you will need to obtain permission directly from the copyright holder. To view a copy of this licence, visit http://creativecommons.org/licenses/by/4.0/. The Creative Commons Public Domain Dedication waiver (http://creativecommons.org/publicdomain/zero/1.0/) applies to the data made available in this article, unless otherwise stated in a credit line to the data. 
Usually, the unbound forms of MoRFs tend to adopt the conformation in the complex [5]. Because of the flexible structure, MoRFs can combine with their partner accurately. Therefore, they play important roles in regulatory processes and signal transduction [6].

MoRFs contain four subtypes: $\alpha$-MoRFs, $\beta$-MoRFs, $\quad$-MoRFs and complex-MoRFs [7]. When MoRFs bond, the four subtypes correspond to $\alpha$-helices, $\beta$-strands, irregular secondary structures and multiple secondary structures respectively. The earliest prediction methods for MoRFs can only predict $\alpha$-MoRFs, such as $\alpha$-MoRF-PredI [8] and $\alpha$-MoRF-PredII [9] based on neural network. Then, a number of methods have emerged to predict all kinds of MoRFs. MoRFpred [10] is the most used comparison prediction method. It contains five types of features which are gained from five disorder predictions [11-14], evolutionary profiles [15], selected amino acid indices [16], predicted B-factors [17] and RSA [18]. Then, a linear kernel support vector machine (SVM) is trained using these features to predict MoRFs. MoRF ${ }_{\mathrm{CHiBi}}[17]$ is a representative method which does not rely on other predictors and evolutionary profiles, but obtains good prediction performance. It trains two SVM based on local physicochemical sequence properties, and combines the outcomes of them to predict MoRFs. MoRF $\mathrm{CHi}_{\mathrm{i}}$ Bi_Light [19] utilizes Bayes rule to combine the scores obtained from ESpritz [20] and $\operatorname{MoRF}_{\mathrm{CHiBi}}$. MoRF $\mathrm{CHiBi}_{\text {_Web }}$ [21] calculates the initial conservation score (ICS) by incorporating three values from the position specific scoring matrixes (PSSM). Then, the prediction results can be obtained by incorporating the ICS and the scores of ESpritz and $\mathrm{MoRF}_{\mathrm{CHiBi}}$. OPAL [22] is also a combined prediction method. It first designs PROMIS [22] through training a SVM model based on half-sphere exposure, solvent accessible surface area and backbone angle information of MoRFs. Finally, OPAL is obtained

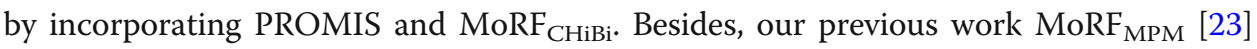
and $\mathrm{MoRF}_{\mathrm{MLP}}$ [24] also obtain good prediction results. MoRF $\mathrm{MPM}_{\text {selects }} 16$ features

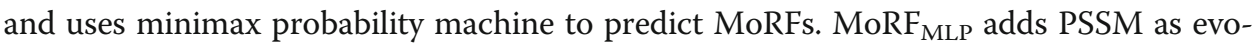
lutionary information to the 16 features selected by $\mathrm{MoRF}_{\mathrm{MPM}}$, and trains MLPs separately for the two kinds of features. Then, their results are fused together to get the final result.

In this paper, we propose a new individual MoRFs prediction method, $\mathrm{MoRF}_{\mathrm{CNN}}$, by training three convolutional neural networks $(\mathrm{CNNs})$ based on three feature sets respectively, and then connecting them together. The first feature set obtains 16 sequence properties from our previous work $M o R F_{M P M}$. The second and third feature sets, derived from $\mathrm{MoRF}_{\mathrm{CHiBi}}$, contain 13 and 14 physicochemical sequence properties respectively. A preprocessing scheme is used to improve the effect of each feature set. Three windows of appropriate length are selected to calculate the features for each residue. Then, they are arranged into a feature matrix for conforming to the input form of $\mathrm{CNN}$. The simulation results show that $\mathrm{MoRF}_{\mathrm{CNN}}$ obtains better performance than other similar prediction methods.

\section{Results}

Datasets

In order to train our prediction method and compare with other methods, we utilize the widely used datasets that are created by Disfani et al. [10] They collect a lot of 
protein complexes concerning interaction between a protein and a small peptide from Protein Data Band [25] of March 2008. These complexes are filtered using a series of principles, and 840 protein sequences are selected. Then, they are divided into TRAINING and TEST sets which contain 421 and 419 protein sequences respectively. After that, using the same protocol, Disfani et al. create another test set TESTNEW which contains 45 protein sequences. To keep up with the comparison methods, we combine TEST and TESTNEW sets into TEST464. Besides, we also utilize TEST_EXP53 set [17] as another independent test set. TEST_EXP53 contains 53 protein sequences and is assembled by Malhis et al. The length of MoRFs in TRAINING and TEST464 sets is between 5 and 25 residues. However, TEST_EXP53 includes 729 MoRF residues from regions with up to 30 residues and 1703 from regions longer than 30 residues. Table 1 lists the specific information.

\section{Performance evaluation}

We mainly utilize ROC (receiver operating characteristic) curve and AUC (the area under the ROC curve) to evaluate the performance. In addition, to evaluate the performance in detail, we also calculate the FPR (the false positive rate) at different TPR (the true positive rate). The FPR and TPR can be denoted as $F P R=T N / N_{\text {non }}, T P R=$ $T P / N_{M o R F}$, where $N_{\text {non }}$ and $N_{\text {MoRF }}$ represent the total number of non-MoRFs and MoRFs residues, $T N$ and $T P$ represent the numbers of accurately predicted MoRFs and non-MoRFs residues, respectively.

\section{Impact of different windows}

In the proposed method, we train three different CNNs based on three feature sets respectively. Based on our previous work, we select three windows for preprocessing with each feature set. The length 10 and 90 windows are used to highlight the characteristics of MoRFs and the surrounding environment, and the length 45 window is used to reduce the noise impact. In this section, we analyze the effect of increasing the number of windows on predictive performance. For comparison, we selected 9 windows in step 10 between windows of length 10 and 90 . The performance of each CNN with 3 windows and 9 windows in TEST set is shown in Fig. 1. The left figures are the full ROC curves of them, and the right figures show their ROC curves at low FPR. Since the number of MoRF residue is much smaller than the number of non-MoRF residue, we will pay more attention to the prediction performance in the low FPR region.

From Fig. 1, the full ROC curves and the ROC curves at low FPR of CNN1, CNN2 and $\mathrm{CNN} 3$ of 3 windows are better than that of 9 windows. The results indicate that selecting too many windows will greatly increase the redundancy in the information,

Table 1 Data sets used in this paper

\begin{tabular}{llllll}
\hline Number & TRAINING & TEST & TESTNEW & TEST464 & TEST_EXP53 \\
\hline Sequences & 421 & 419 & 45 & 464 & 53 \\
MoRFs Residues & 5396 & 5153 & 626 & 5779 & 2432 \\
non-MoRFs Residues & 240,588 & 253,676 & 36,907 & 290,583 & 22,754 \\
Total Residues & 245,984 & 258,829 & 37,533 & 296,362 & 25,186 \\
\hline
\end{tabular}




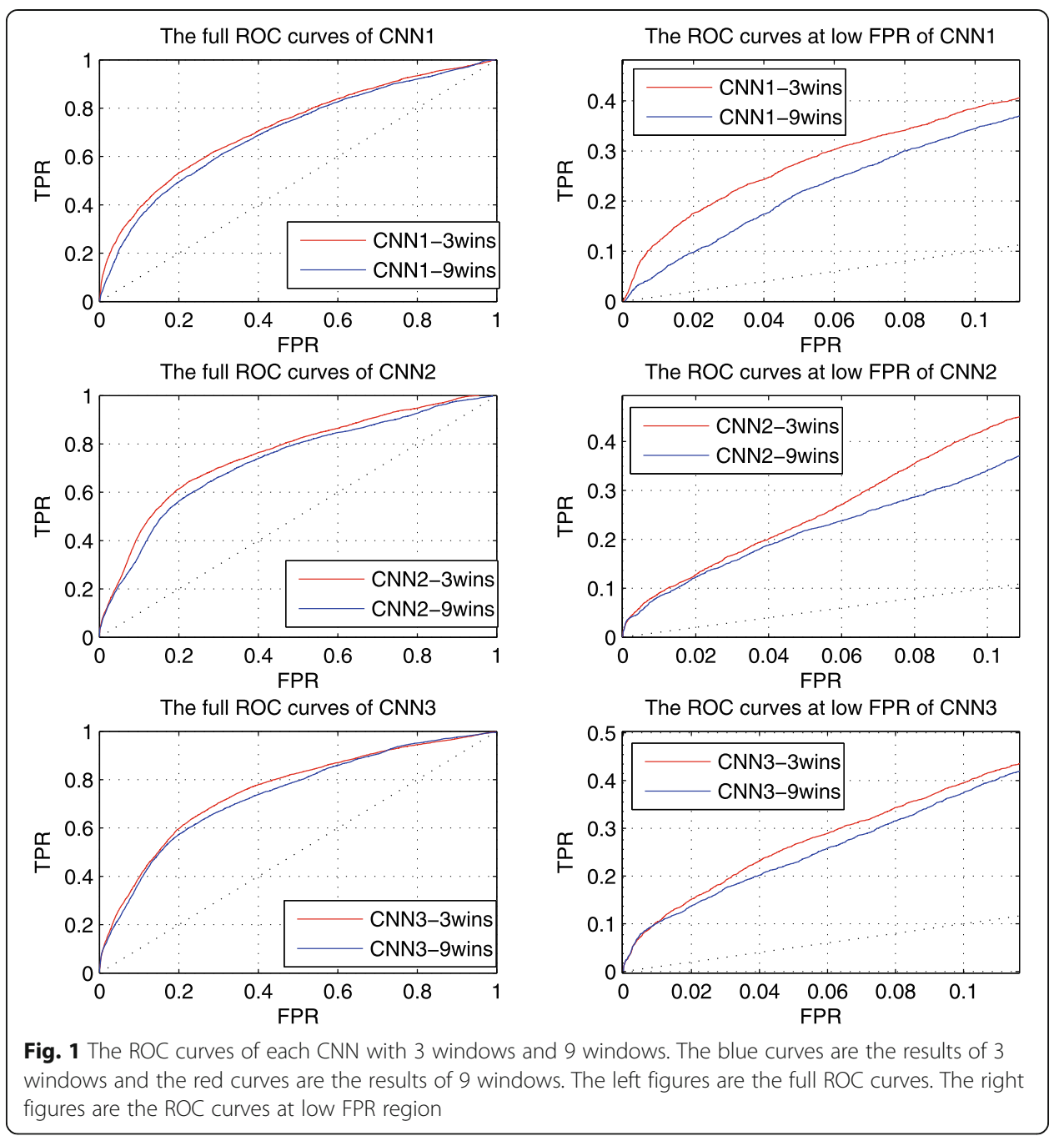

and thus increase the noise in the feature matrix. Therefore, only 3 windows with length of 10, 45 and 90 are selected for preprocessing and feature matrix calculation.

\section{Impact of different activation functions}

In this section, we compare the effects of different activation functions of each convolutional layer on the prediction performance. Figure 2 shows the prediction performance of ReLu function, sigmoid function and hyperbolic tangent function based on the third feature sets in TEST set.

From Fig. 2, the full ROC curve and the ROC curve at low FPR of ReLu function are similar to that of hyperbolic tangent function. However, the performance of sigmoid function is significantly worse. Thus, we select ReLu function as the activation function.

\section{Comparing CNNs and their combination}

In this section, we compare the prediction performance of each $\mathrm{CNN}$ and the prediction performance of combining the prediction results of $\mathrm{CNN}$ directly. Figure 3 shows 

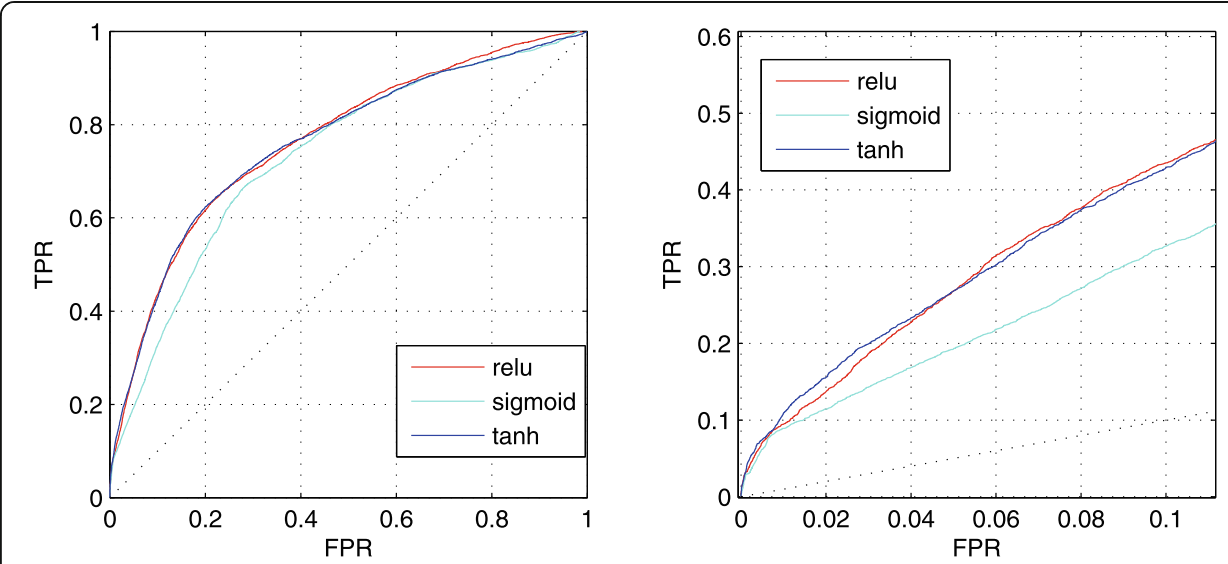

Fig. 2 The ROC curves of CNN3 with different activation functions. The left figure is the full ROC curves. The right figure is the ROC curves at low FPR region

the prediction performance of them in TEST set. The left figure is the full ROC curves of them, and the right figure shows their ROC curves at low FPR. The red curves describe the average values of the prediction results of three CNNs. Through averaging, prediction performance improves a bit on both the full ROC curve and the ROC curve at low FPR.

\section{Impact of different convolutional layers}

We change the number of convolutional layers to analyze the influence on the prediction performance. Figure 4 shows the prediction performance of the combined results of three CNNs in TEST set with different convolutional layers.

From Fig. 4, the performance of 3 layers is similar to that of 2 layers. Besides, as the number of convolutional layers continues to increase, the prediction performance does not improve. Therefore, we still choose two convolutional layers for prediction.

\section{Comparing with other prediction methods}

In this section, we compare our method, $M o R F_{C N N}$, with MoRFpred, MoRF $F_{C H i B i}$, MoRF $F_{\text {CHiBi_Light }}$ and MoRF $F_{M P M}$. Among these methods, MoRFpred is a classical
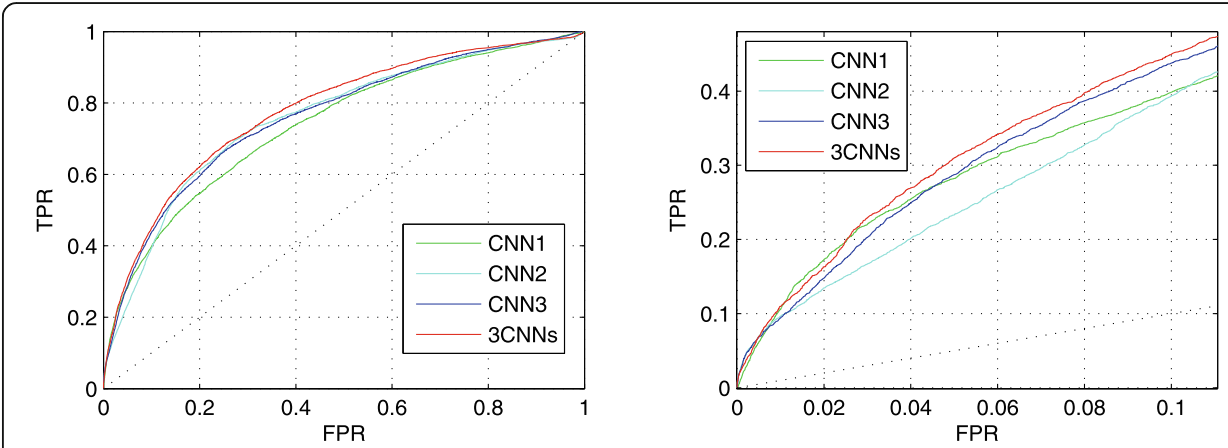

Fig. 3 The ROC curves of three CNNs and their combined result. The red curves describe the combination result. The left figure shows the full ROC curves. The right figure shows the ROC curves at low FPR region 

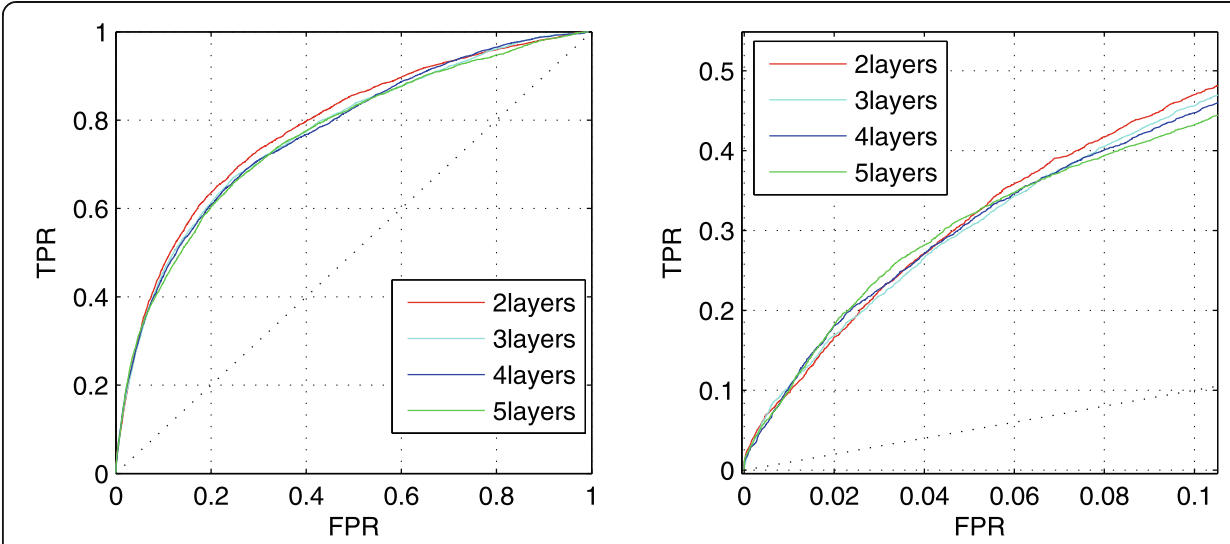

Fig. 4 The ROC curves of the combined results with different convolutional layers. The left figure describes the full ROC curves. The right figure describes the ROC curves at low FPR region

method, $\mathrm{MoRF}_{\mathrm{CHiBi}}$ and $\mathrm{MoRF}_{\mathrm{MPM}}$ are individual methods and do not use evolutionary information, MoRF $\mathrm{CHiBi}_{\text {Light }}$ combines the scores of ESpritz and MoRF $\mathrm{CH}_{\mathrm{CHBi}}$. Because $\mathrm{MoRF}_{\mathrm{CNN}}$ is a new individual MoRFs prediction method without evolutionary information, it is compared with similar types of methods. We use TEST464 and TEST_EXP53 sets for the performance comparison. Table 2 shows the AUC values of $\mathrm{MoRF}_{\mathrm{CNN}}$ and other methods. From Table 2, MoRF ${ }_{\mathrm{CNN}}$ gets higher AUC than MoRFpred, MoRF $\mathrm{CHiBi}_{\text {, }}$ $\mathrm{MoRF}_{\mathrm{CHiBi} \_ \text {Light }}$ and MoRF $\mathrm{MPM}_{\mathrm{M}}$ on both TEST464 and TEST_EXP53 sets. In addition, $\mathrm{MoRF}_{\mathrm{CNN}}$ can process about 9000 residues per minute, which is similar to MoRF $_{\text {CHiBi_Light. }}$.

We also compute the FPR values at different TPR to further analyze the performance of our method, as shown in Table 3. Obviously, $\mathrm{MoRF}_{\mathrm{CNN}}$ obtains lower FPR values than MoRFpred as well as $\mathrm{MoRF}_{\mathrm{CHiBi}}$, and obtains similar FPR values to MoRF $\mathrm{CHiBi}_{-}$ Light and MoRF ${ }_{\mathrm{MPM}}$.

\section{Discussion}

The proposed method MoRF $\mathrm{CNN}_{\mathrm{N}}$ is an individual MoRFs prediction method which just uses protein sequence properties. These protein sequence properties are divided into three feature sets. The first feature set is from $\mathrm{MoRF}_{\mathrm{MPM}}$ containing 13 physicochemical properties, 2 disorder propensities and topological entropy. The second and third feature sets, derived from $\mathrm{MoRF}_{\mathrm{CHiBi}}$, contain 13 and 14 physicochemical properties respectively. To highlight the relationship between the residue and its surrounding environment, three windows are utilized to preprocess these three feature sets. Then, the preprocessed features are arranged into a feature matrix conforming to the input form of $\mathrm{CNN}$. We train three CNNs based on three feature sets respectively, and then combine their results together. The simulation results show that $M_{\text {oRF }}{ }_{\mathrm{CNN}}$ is effective and competitive.

Table 2 AUC On TEST464 and TEST_EXP53

\begin{tabular}{llllll}
\hline & MoRF $_{\text {CNN }}$ & MoRFpred & MoRF $_{\text {CHiBi }}$ & MoRF $_{\text {CHiBi_Light }}$ & MoRF $_{\text {MPM }}$ \\
\hline TEST464 & $\mathbf{0 . 7 8 7}$ & 0.675 & 0.743 & 0.777 & 0.778 \\
TEST_EXP53 & $\mathbf{0 . 8 0 1}$ & 0.620 & 0.712 & 0.799 & 0.758 \\
\hline
\end{tabular}


Table 3 FPR at different TPR on TEST464 and TEST_EXP53

\begin{tabular}{|c|c|c|c|c|c|c|}
\hline & \multicolumn{2}{|l|}{ TPR $=0.2$} & \multicolumn{2}{|l|}{$\mathrm{TPR}=0.3$} & \multicolumn{2}{|l|}{$\mathrm{TPR}=0.4$} \\
\hline & TEST464 & TEST_EXP53 & TEST464 & TEST_EXP533 & TEST464 & TEST_EXP533 \\
\hline $\operatorname{MoRF}_{C N N}$ & 0.026 & 0.025 & 0.045 & 0.041 & 0.072 & 0.073 \\
\hline MoRFpred & 0.033 & 0.083 & 0.071 & 0.146 & 0.143 & 0.221 \\
\hline $\mathrm{MoRF}_{\mathrm{CH} \mathrm{iBi}}$ & 0.031 & 0.031 & 0.063 & 0.064 & 0.104 & 0.125 \\
\hline MoRF ${ }_{\text {CHiBi_Light }}$ & 0.020 & 0.016 & 0.040 & 0.043 & 0.073 & 0.068 \\
\hline $\mathrm{MoRF}_{\mathrm{MPM}}$ & 0.027 & 0.025 & 0.047 & 0.056 & 0.074 & 0.096 \\
\hline
\end{tabular}

The following points enable $\mathrm{MoRF}_{\mathrm{CNN}}$ to obtain good performance. First, the three feature sets of protein sequence properties are effective for predicting MoRFs. Second, the preprocessing process enhances the performance of these selected properties. Third, the constructed CNN prediction model can reflect the relationship between each feature and its neighboring features in the protein feature matrix, and find out more information from different features, and thus enrich the information proposed by protein sequences.

\section{Conclusions}

In this paper, we propose a new individual MoRFs prediction method, $M o R F_{\mathrm{CNN}}$, based on sequence properties and convolutional neural networks. Comparing with other

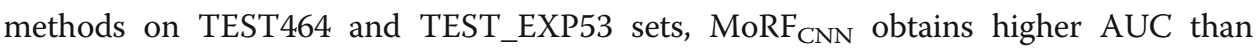
MoRFpred, MoRF $F_{\mathrm{CHiBi}}, \mathrm{MoRF}_{\mathrm{CHiBi} \_ \text {Light }}$ and $\mathrm{MoRF}_{\mathrm{MPM}}$. In addition, MoRF $\mathrm{CNN}_{\mathrm{C}}$ achieves lower FPR than MoRFpred and $\mathrm{MoRF}_{\mathrm{CHiBi}}$, as well as similar FPR to MoRF $\mathrm{CHiBi}_{\text {LLight }}$ and $\mathrm{MoRF}_{\mathrm{MPM}}$ when TPR is set to $0.2,0.3$ and 0.4. In the future, we will research different combination of the feature matrix and modify the topological structure of $\mathrm{CNN}$ to further improve the prediction performance.

\section{Methods}

\section{Feature selection}

We select three feature sets to describe the properties of MoRFs in this paper. The first feature set obtains 16 sequence properties which are from our previous work MoRF $_{\text {MPM. }}$. This feature set includes 13 physicochemical properties, 2 disorder propensities and topological entropy. Among them, the 13 physicochemical properties are selected from Amino Acid Index [16] using simulated annealing algorithm, the 2 disorder propensities are the Remark 465 and Deleage/Roux from GlobPlot NAR paper [26], the topological entropy is calculated after mapping the protein sequence to 0-1 sequence [27]. The second and third feature sets, derived from $\mathrm{MoRF}_{\mathrm{CHiBi}}$, contain 13 and 14 physicochemical sequence properties from Amino Acid Index respectively.

In order to highlight the effect of these feature sets, we preprocess protein sequences according to each feature set. Taking the first feature set as an example, for a general protein sequence $w w$ with length $L$, we select a window with the length of $N(N<L)$ and fill $N_{0}=\llcorner(N-1) / 2\rfloor$ zeros at the beginning and end of the sequence. Then, the sequence length becomes $L_{0}=L+2 N_{0}$. We slide the window 
to intercept regions of length $N$ with step of 1 . For each intercept region, topological entropy is calculated through Eq. 14 of [27], and the remaining 15 sequence properties are calculated by the average value of mapped region of these properties. The calculated 16 dimensional vector $\mathbf{v}_{i}(1 \leq i \leq L)$ is assigned to each residue in the region. After that, as the window slides, the vectors obtained by each residue are accumulated, and the average value is taken as the final feature vector for each residue under this window. This process can be represented as

$$
\boldsymbol{x}_{j}=\left\{\begin{array}{l}
\frac{1}{j+N_{0}} \sum_{i=1}^{j+N_{0}} \mathbf{v}_{i}, 1 \leq j \leq N_{0} \\
\frac{1}{N} \sum_{i=j+N_{0}-N+1}^{j+N_{0}} \mathbf{v}_{i}, N_{0}<j \leq L-N_{0} \\
\frac{1}{L_{0}-j-N_{0}+1} \sum_{i=j+N_{0}-N+1}^{L_{0}-N+1} \mathbf{v}_{i}, L-N_{0}<j \leq L
\end{array}\right.
$$

We can get a 16 dimensional feature vector for each residue under one window. In this paper, we choose several windows to preprocess. In order to conform to the input characteristics of $\mathrm{CNN}$, we combine the feature vectors calculated from different windows into a feature matrix for each residue. Then, each residue can obtain a $N_{w i n} \times 16$ feature matrix for the first feature set, where $N_{\text {win }}$ denotes the number of windows. Similarly, each residue can obtain $N_{\text {win }} \times 13$ and $N_{\text {win }} \times 14$ feature matrices for the second and third feature sets.

Based on our previous work, we select three windows of length 10, 45, and 90 for preprocessing. Among them, the short window is used to highlight the characteristics of MoRFs, the long window is used to highlight the characteristics of MoRFs surrounding environment, and the middle window is used to reduce the noise impact brought by the long window.

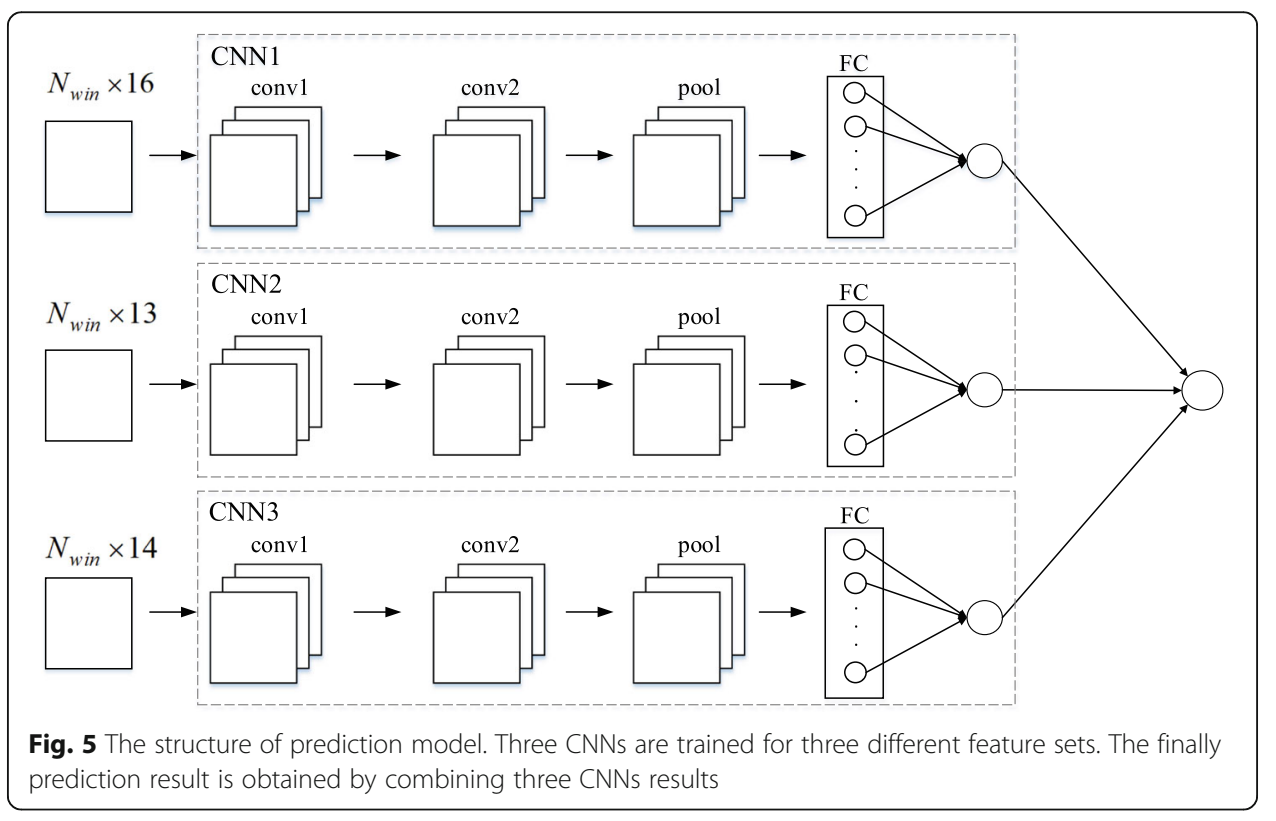




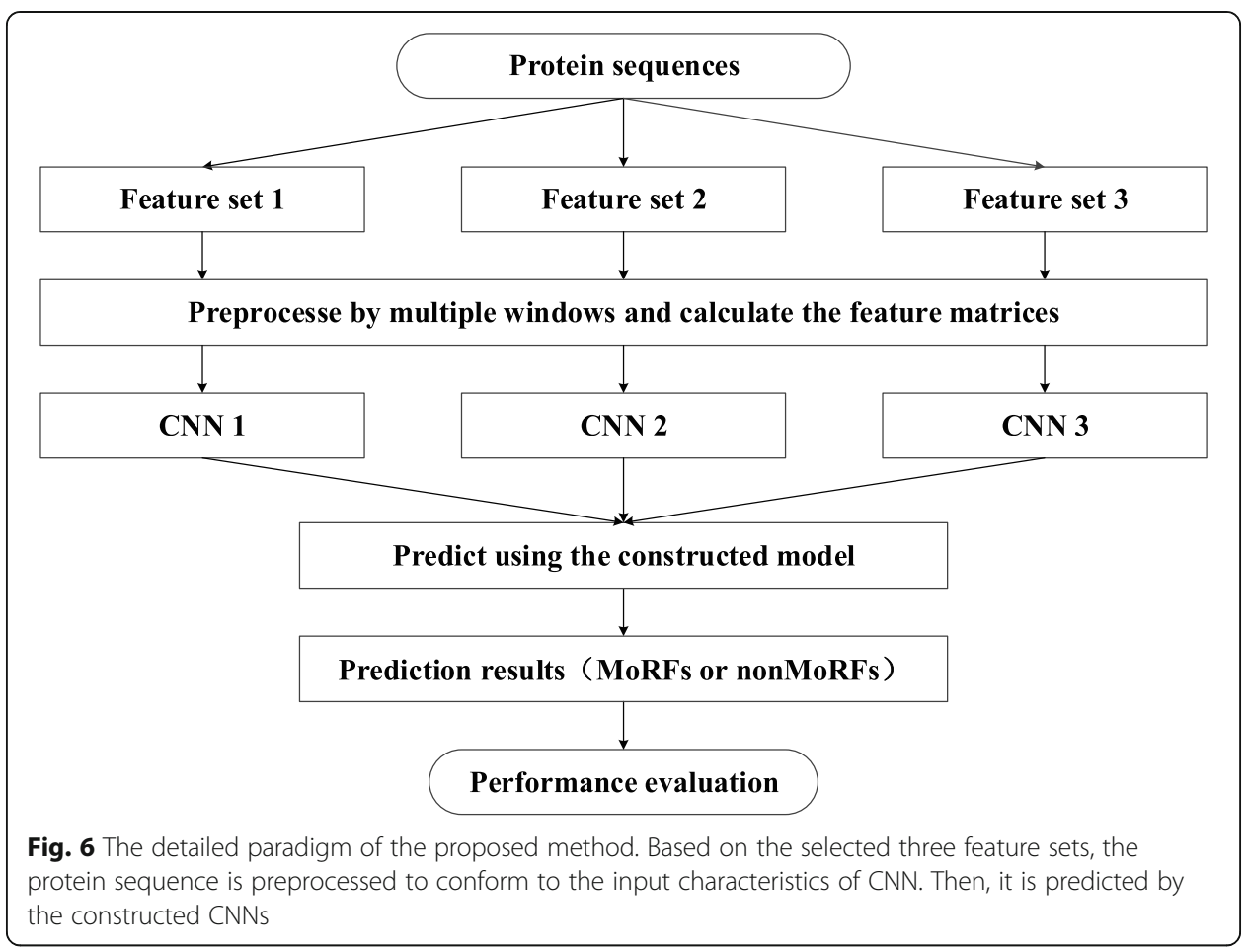

\section{Prediction model}

We utilize the TRAINING set to train our prediction model. Three CNNs (CNN1, $\mathrm{CNN} 2$ and CNN3) are trained based on the selected three feature sets respectively. The finally prediction result is obtained by the average values of three CNNs results. Figure 5 shows the structure of prediction model.

Each $\mathrm{CNN}$ contains two convolutional layers and one pooling layer as well as one fully connected layer. The activation function of each convolutional layer is ReLu function, and the activation function of the output layer is sigmoid function. In each convolution layer, the convolution step is 1 and performs same padding with zero. The parameters of conv1 and conv2 are set to $2 \times 2 \times 1 \times 16$ and $2 \times 2 \times 16 \times 8$ respectively. The pooling layer uses max pooling with $2 \times 2$ filter. In the designed $\mathrm{CNN}$, the gradient descent algorithm is replaced by Adam algorithm [28] in the backward propagation to update parameters. In order to improve the operation speed, mini-batch is used to update parameters. That is, the sample set is divided into multiple subsets of equal scale for the each iteration, and each subset is used to calculate the gradient and update parameters one by one. In order to present our method more visually, combined with the feature selection, Fig. 6 shows the detailed paradigm of the proposed method.

\section{Abbreviations}

MoRF: molecular recognition feature; CNN: convolutional neural network; IDP: intrinsically disordered protein; SVM: support vector machine; ICS: initial conservation score; PSSM: position specific scoring matrixes; ROC: receiver operating characteristic; AUC: the area under the ROC curve; FPR: the false positive rate; TPR: the true positive rate 


\section{Funding}

This work was supported by Hebei Province University Science and Technology Research Project (No.QN2021038), the Sub-Project of Intelligent Robot under National Key R\&D Program of China (No.2019YFB1312102), Hebei Province Natural Science Foundation (No.F2019202364), National Natural Science Foundation of China (No. 61801164). The funding bodies have no role in the design of the study and collection, analysis, and interpretation of data and in writing the manuscript.

Availability of data and materials

The datasets supporting the conclusions of this article are available on the references [10,29].

\section{Declarations}

\section{Ethics approval and consent to participate}

Not applicable.

\section{Consent for publication}

Not applicable.

\section{Competing interests}

The authors declare that they have no competing interests.

Received: 13 February 2021 Accepted: 8 August 2021

Published online: 14 August 2021

\section{References}

1. Necci M, Piovesan D, Dosztányi Z, Tompa P, Tosatto SCE. A comprehensive assessment of long intrinsic protein disorder from the DisProt database. Bioinformatics. 2018;34(3):445-52. https://doi.org/10.1093/bioinformatics/btx590.

2. Liu Y, Wang X, Liu B. RFPR-IDP: reduce the false positive rates for intrinsically disordered protein and region prediction by incorporating both fully ordered proteins and disordered proteins. Brief Bioinform. 2020;00:1-12.

3. Sharma R, Sharma A, Patil A, Tsunoda T. Discovering MoRFs by trisecting intrinsically disordered protein sequence into terminals and middle regions. BMC Bioinformatics. 2019;19(S13):378. https://doi.org/10.1186/s12859-018-2396-7.

4. Cumberworth A, Lamour G, Babu MM, Gsponer J. Promiscuity as a functional trait: intrinsically disordered regions as central players of interactomes. Biochem J. 2013;454(3):361-9. https://doi.org/10.1042/BJ20130545.

5. Dunker AK, Bondos SE, Huang F, Oldfield CJ. Intrinsically disordered proteins and multicellular organisms. Semin Cell Dev Biol. 2015;37:44-55. https://doi.org/10.1016/j.semcdb.2014.09.025.

6. Staneva I, Huang Y, Liu Z, Wallin S. Binding of two intrinsically disordered peptides to a multi-specific protein: a combined Monte Carlo and molecular dynamics study. PLoS Comput Biol. 2012;8(9):e1002682. https://doi.org/10.1371/ journal.pcbi.1002682.

7. Lee R, Buljan M, Lang B, Weatheritt RJ, Daughdrill GW, Dunker AK, et al. Classification of intrinsically disordered regions and proteins. Chem Rev. 2014;114(13):6589-631. https://doi.org/10.1021/cr400525m.

8. Oldfield CJ, Cheng Y, Cortese MS, Romero P, Uversky VN, Dunker AK. Coupled folding and binding with alpha-helixforming molecular recognition elements. Biochemistry. 2005;44(37):12454-70. https://doi.org/10.1021/bi050736e

9. Cheng Y, Oldfield CJ, Meng J, Romero P, Uversky VN, Dunker AK. Mining a-helix-forming molecular recognition features with cross species sequence alignments. Biochemistry. 2007;46(47):13468-77. https://doi.org/10.1021/bi7012273.

10. Disfani FM, Hsu WL, Mizianty MJ, Oldfield CJ, Xue B, Dunker AK, et al. MoRFpred, a computational tool for sequencebased prediction and characterization of short disorder-to-order transitioning binding regions in proteins. Bioinformatics. 2012;28(12):i75-83. https://doi.org/10.1093/bioinformatics/bts209.

11. Dosztányi Z, Csizmok V, Tompa P, Simon I. IUPred: web server for the prediction of intrinsically unstructured regions of proteins based on estimated energy content. Bioinformatics. 2005;21(16):3433-4. https://doi.org/10.1093/bioinformatics/ bti541.

12. Ward JJ, LJ MG, Bryson K, Buxton BF, Jones DT. The DISOPRED server for the prediction of protein disorder. Bioinformatics. 2004;20(13):2138-9. https://doi.org/10.1093/bioinformatics/bth195.

13. McGuffin $\sqcup$. Intrinsic disorder prediction from the analysis of multiple protein fold recognition models. Bioinformatics. 2008;24(16):1798-804. https://doi.org/10.1093/bioinformatics/btn326.

14. Mizianty MJ, Stach W, Chen K, Kedarisetti KD, Disfani FM, Kurgan L. Improved sequence-based prediction of disordered regions with multilayer fusion of multiple information sources. Bioinformatics. 2010;26(18):i489-96. https://doi.org/10.1 093/bioinformatics/btq373.

15. Altschul SF, Madden TL, Schäffer AA, Zhang J, Zhang Z, Miller W, et al. Gapped BLAST and PSI-BLAST: a new generation of protein database search programs. Nucleic Acids Res. 1997;25(17):3389-402. https://doi.org/10.1093/nar/25.17.3389.

16. Kawashima S, Pokarowski P, Pokarowska M, Kolinski A, Katayama T, Kanehisa M. AAindex: amino acid index database, progress report 2008. Nucleic Acids Res. 2008;36(Database issue):D202-5. https://doi.org/10.1093/nar/gkm998.

17. Schlessinger A, Yachdav G, Rost B. PROFbval: predict flexible and rigid residues in proteins. Bioinformatics. 2006;22(7): 891-3. https://doi.org/10.1093/bioinformatics/btl032.

18. Faraggi $E$, Xue B, Zhou Y. Improving the prediction accuracy of residue solvent accessibility and real-value backbone torsion angles of proteins by fast guided learning through a two-layer neural network. Proteins. 2009;74(4):847-56. https://doi.org/10.1002/prot.22193.

19. Malhis N, Jacobson M, Gsponer J. MoRFchibi system: software tools for the identification of MoRFs in protein sequences. Nucleic Acids Res. 2016;44:488-93.

20. Walsh,l, Martin AJM, Domenico TD, Tosatto SCE. ESpritz: accurate and fast prediction of protein disorder. Bioinformatics 2012;28:503-509 
21. Malhis N, Wong ETC, Nassar R, Gsponer J. Computational identification of MoRFs in protein sequences using hierarchical application of Bayes rule. PLoS One. 2015;10(10):e0141603. https://doi.org/10.1371/journal.pone.0141603.

22. Sharma R, Raicar G, Tsunoda T, Patil A, Sharma A. OPAL: prediction of MoRF regions in intrinsically disordered protein sequences. Bioinformatics. 2018;34(11):1850-8. https://doi.org/10.1093/bioinformatics/bty032.

23. He H, Zhao J, Sun G. Computational prediction of MoRFs based on protein sequences and minimax probability machine. BMC Bioinformatics. 2019;20(1):529. https://doi.org/10.1186/s12859-019-3111-z.

24. He H, Zhao J, Sun G. Prediction of MoRFs in protein sequences with MLPs based on sequence properties and evolution information. Entropy. 2019;21(7):635. https://doi.org/10.3390/e21070635.

25. Berman H, Henrick K, Nakamura H, Markley JL. The worldwide protein data Bank (wwPDB): ensuring a single, uniform archive of PDB data. Nucleic Acids Res. 2007;35(Database):D301-3. https://doi.org/10.1093/nar/gkl971.

26. Linding R, Russell RB, Neduva V, Gibson TJ. Globplot: exploring protein sequences for globularity and disorder. Nucleic Acids Res. 2003;31(13):3701-8. https://doi.org/10.1093/nar/gkg519.

27. He H, Zhao JX. A low computational complexity scheme for the prediction of intrinsically disordered protein regions. Math Probl Eng. 2018;2018:1-7. https://doi.org/10.1155/2018/8087391.

28. Kingma DP, Adam JB. A method for stochastic optimization. CoRR. 2015;1412:6980.

29. Malhis N, Gsponer J. Computational identification of MoRFs in protein sequences. Bioinformatics. 2015;31(11):1738-44. https://doi.org/10.1093/bioinformatics/btv060.

\section{Publisher's Note}

Springer Nature remains neutral with regard to jurisdictional claims in published maps and institutional affiliations.

Ready to submit your research? Choose BMC and benefit from:

- fast, convenient online submission

- thorough peer review by experienced researchers in your field

- rapid publication on acceptance

- support for research data, including large and complex data types

- gold Open Access which fosters wider collaboration and increased citations

- maximum visibility for your research: over $100 \mathrm{M}$ website views per year

At $\mathrm{BMC}$, research is always in progress.

Learn more biomedcentral.com/submissions 